ECG-based prediction of atrial fibrillation development following coronary artery bypass grafting

This article has been downloaded from IOPscience. Please scroll down to see the full text article.

2010 Physiol. Meas. 31663

(http://iopscience.iop.org/0967-3334/31/5/005)

The Table of Contents and more related content is available

Download details:

IP Address: 128.178.83.57

The article was downloaded on 24/03/2010 at 10:42

Please note that terms and conditions apply. 


\title{
ECG-based prediction of atrial fibrillation development following coronary artery bypass grafting
}

\author{
Sinisa Sovilj ${ }^{1,4}$, Adriaan Van Oosterom ${ }^{2}$, Gordana Rajsman ${ }^{3}$ and \\ Ratko Magjarevic ${ }^{1}$ \\ ${ }^{1}$ Faculty of Electrical Engineering and Computing, University of Zagreb, 10000 Zagreb, Croatia \\ ${ }^{2}$ Department Cardiomet, Centre Hospitalier Universitaire Vaudois, 1011 Lausanne, Switzerland \\ ${ }^{3}$ Department of Cardiac Surgery, Clinical Hospital Center Zagreb, 10000 Zagreb, Croatia \\ E-mail: sinisa.sovilj@ieee.org
}

Received 4 January 2010, accepted for publication 19 February 2010

Published 22 March 2010

Online at stacks.iop.org/PM/31/663

\begin{abstract}
In patients undergoing coronary artery bypass grafting (CABG) surgery, postoperative atrial fibrillation (AF) occurs with a prevalence of up to $40 \%$. The highest incidence is seen between the second and third day after the operation. Following cardiac surgery AF may cause various complications such as hemodynamic instability, heart attack and cerebral or other thromboembolisms. AF increases morbidity, duration and expense of medical treatments. This study aims at identifying patients at high risk of post-operative AF. Early prediction of AF would provide timely prophylactic treatment and would reduce the incidence of arrhythmia. Patients at low risk of post-operative AF could be excluded on the basis of the contraindications of anti-arrhythmic drugs. The study included 50 patients in whom lead II electrocardiograms were continuously recorded for $48 \mathrm{~h}$ following CABG. Univariate statistical analysis was used in the search for signal features that could predict AF. The most promising ones identified were $P$ wave duration, RR interval duration and PQ segment level. On the basis of these, a nonlinear multivariate prediction model was made by deploying a classification tree. The prediction accuracy was found to increase over time. At $48 \mathrm{~h}$ following $\mathrm{CABG}$, the measured best smoothed sensitivity was $84.8 \%$ and the specificity $85.4 \%$. The positive and negative predictive values were $72.7 \%$ and $92.8 \%$, respectively, and the overall accuracy was $85.3 \%$. With regard to the prediction accuracy, the risk assessment
\end{abstract}

4 Author to whom any correspondence should be addressed. 
and prediction of post-operative AF is optimal in the period between 24 and $48 \mathrm{~h}$ following CABG.

Keywords: post-operative atrial fibrillation, $\mathrm{CABG}, P$ wave onset trigger, cubic spline baseline correction, PQ segment level, classification tree

(Some figures in this article are in colour only in the electronic version)

\section{Introduction}

Atrial fibrillation (AF) is the most common supraventricular arrhythmia and post-operative complication. In patients after coronary artery bypass grafting surgery (CABG), the prevalence is $30-40 \%$. Patients who develop post-operative AF typically do not have a previous history of AF. The onset of AF most frequently occurs between the second and the third day after surgery.

Commonly assumed electrophysiological mechanisms inducing AF are the slow propagation velocity of atrial impulses, inhomogeneous atrial activation and shortening of atrial refractory period. The etiology is not entirely clarified. It is presumed that AF is induced by post-operative shock, ischemia or inflammation of the atrium (Stewart et al 2001, Kannel et al 1998, Petrač 1996).

AF is associated with numerous clinical complications, leads to hemodynamic instability, raises the risk of thromboembolic incidence and increases morbidity, as well as the duration and cost of hospitalization (Hakala and Hedman 2003, Hashimoto et al 1991, Creswell et al 1993, Borzak et al 1998).

The aim of this study is the development of a prediction model for the identification of patients at high risk of AF following CABG. Prediction of AF prior to the onset of AF could enable more appropriate, effective or better-adjusted prophylactic therapy.

Earlier studies have attempted to find possible predictors of post-operative AF in different demographics and clinical risk factors observed pre-operatively, intra-operatively and postoperatively (table 1). The results of the applied multivariate statistical models, such as logistic regression, which comprised multiple pre-operative and intra-operative risk factors, did not significantly improve discrimination and AF prediction. In fact, none of the demographic or clinical risk factors attained a sufficient significance level for implementation in clinical practice as an independent predictor of post-operative AF (Hakala and Hedman 2003).

Prediction of post-operative AF derived from the ECG is usually based on signal averaged $P$ wave ECG (SAECG), standard ECG leads II or V1 and heart rate variability (HRV). The results are controversial.

A comparison of the results from different studies on the prediction of post-operative AF based on the ECG is shown in table 2. These studies were performed on populations in which all patients underwent CABG. Some studies with similar patients' data compared patients before and after CABG surgery (Andrikopoulos et al 2000, Aytemir et al 2000, Dilaveris et al 1998, Fukunami et al 1991). Other studies compared patients prone to AF and a control group of healthy subjects (Klein et al 1995, Steinberg et al 1993). The results of these studies cannot be compared with the quality of prediction of post-operative AF based on patients' data obtained only after CABG. 
Table 1. Demographic and clinical risk factors and potential AF predictors.

Pre-operative

Age, gender, pervious AF history, hypertension, stenosis of right coronary artery, chronic obstructive pulmonary disease (COPD), use of digoxin, $\beta$-blocking withdrawal effect, previous myocardial infraction, smoking, diabetes mellitus, left ventricular ejection fraction (LVEF), left atrium dimension

Intra-operative

Aortic cross-clamp time, graft number, graft position, choice of alternative minimal invasive off-pump CABG versus conventional on-pump CABG

Post-operative

Mechanical ventilation time, post-operative pneumonia, assistance of intra-aortic balloon pump, total amount of administrated fluids

Table 2. Comparison of the results from studies on the prediction of post-operative AF based on the ECG.

\begin{tabular}{llllllll}
\hline Study & Signal & Sn $(\%)$ & Sp $(\%)$ & PP $(\%)$ & NP $(\%)$ & Acc $(\%)$ & AF $/ N$ \\
\hline Buxton et al 1981 & II lead & 66 & 70 & 48 & 83 & 68.8 & $29 / 99$ \\
Zaman et al 1997 & SAECG & 63 & 74 & 49 & 84 & 70.9 & $16 / 64$ \\
Stafford et al 1997 & SAECG & 73 & 48 & 34 & 83 & 54.7 & $51 / 189$ \\
Aytemir et al 1999 & SAECG & 68 & 88 & 76 & 83 & 80.8 & $19 / 53$ \\
Chang et al 1999 & II lead & - & - & 37 & 83 & 68.8 & $37 / 120$ \\
Passman et al 2001 & V1 lead & 58 & 70 & 39 & 83 & 67.1 & $64 / 152$ \\
Caravelli et al 2002 & SAECG & 84 & 73 & 85 & 70 & 84.6 & $56 / 129$ \\
Vassilikos et al 2003 & SAECG & 91 & 65 & - & - & 73.8 & $17 / 50$ \\
Present study & II lead & 85.8 & 85.4 & 72.7 & 92.8 & 85.3 & $14 / 50$ \\
\hline
\end{tabular}

$\mathrm{Sn}=$ sensitivity, $\mathrm{Sp}=$ specificity, $\mathrm{PP}=$ positive predictive value, $\mathrm{NP}=$ negative predictive value Acc $=$ accuracy, $\mathrm{AF} / \mathrm{N}=\mathrm{AF}$ group size and overall size.

\section{Methods}

\subsection{Data acquisition}

Lead II signals of the standard 12-lead ECG were recorded, continuously, for $48 \mathrm{~h}$ following CABG. The length of the recording restricted the acquisition to a single lead. Lead II was chosen since atrial activity is clearly observable in this lead. ECG acquisition was made by means of the HP patient monitor 78330A and an ADC card (measurement computing CIODAS08/JR). The sampling frequency was $1000 \mathrm{~Hz}$, with the voltage resolution of $0.488 \mu \mathrm{V}$. In parallel, the patients were monitored and AF was detected separately by the Philips CMS patient monitoring system.

In most previous studies, ECG recordings over episodes were lasting just a few minutes, while generally not specifying the time of ECG acquisition with reference to the termination of CABG procedure. In our approach high-resolution ECG monitoring was performed over an extended episode (up to $48 \mathrm{~h}$ ) in the search for potential trends in the quality of prediction. The inclusion of the pre-operative, operative and post-operative clinical factors could enhance the predictive model, but our study was principally focused on the ECG-based prediction of AF only. 
Table 3. Study patients.

\begin{tabular}{llll}
\hline Group & Male & Female & Age \\
\hline AF group & $58 \%$ & $42 \%$ & $65.9 \pm 3.5$ \\
SR group & $72 \%$ & $28 \%$ & $60.9 \pm 6.4$ \\
Population & $70 \%$ & $30 \%$ & $64.8 \pm 6.3$ \\
\hline
\end{tabular}

\subsection{Study patients}

The patients with no prior arrhythmia history underwent a conventional on-pump CABG, with typically two or three bypassed vessels. The acquisition of post-operative ECG was approved from the Clinical Hospital Center Zagreb and the patients were informed. Data were collected from 50 patients, of whom 14 patients $(28 \%)$ developed AF during or after the recording, and 36 patients $(72 \%)$ did not develop AF, neither during nor after the recording. AF was defined as fibrillation or flutter lasting more than $1 \mathrm{~h}$ and was determined accurately by skilled personnel. For the patients who developed AF during the recording episode, only the part of ECG during sinus rhythm, until the onset of AF, was included in the analysis.

The patient population consisted of $70 \%$ males and $30 \%$ females, of mean age $64.8 \pm$ 6.3. The group who developed AF post-operatively (AF group) consisted of $58 \%$ males, $42 \%$ females, of mean age $65.9 \pm 3.5$. The patients who maintained sinus rhythm (SR group) consisted of $72 \%$ males, $28 \%$ females, of mean age $60.9 \pm 6.4$ (table 3 ). The age difference between the groups was examined and was not found significantly different.

The pre-operative left ventricular ejection fraction was spread between $45 \%$ and $60 \%$. No evidence for the post-operative pericarditis was found. Beta-blockers were routinely administrated to decrease cardiac workload and oxygen demand, but were not used as a prophylactic against arrhythmias, while the anti-arrhythmic drugs were administered only if AF with the very rapid ventricular rate occurred.

\subsection{Data processing}

The electrocardiograms were segmented using a custom-made QRS and $P$ wave detector based on a dyadic wavelet decomposition of ECG, which features robust detection, high accuracy and precision in determination of onset, peak and ending of respective waves (Li et al 1995). The accuracy of custom-made QRS detection was tested on the standard MIT-BIH Arrhythmia Database of annotated ECG signals. A reproducible high sensitivity of $99.70 \%$ and a high positive predictive value of $99.73 \%$ of a custom-made QRS detector were found.

For $P$ wave detection and segmentation, the algorithm identifies five characteristic $P$ wave markers: timing onset (Ponset), magnitude of apex (Peak), ending or offset (Poffset), maximal up-slope (Pslope1) and maximal down-slope (Pslope2) (Sovilj et al 2005, Li et al 1995). These points were used for the subsequent measurement of different $P$ wave features and their trends (figure 1).

An important aspect of ECG processing is the identification of the baseline and the subsequent correction of its drift, which is the essential condition for the accurate and precise measurement of amplitudes in any part of ECG (level of the PQ segment, $P$ wave amplitude, etc). Estimation and correction were made by using the cubic spline baseline interpolation through the onsets of the $P$ waves (Ponset) of the successive beats. $P$ wave onset is the best point for interpolation since it is the most stable point in the ECG (Ihara et al 2006). 

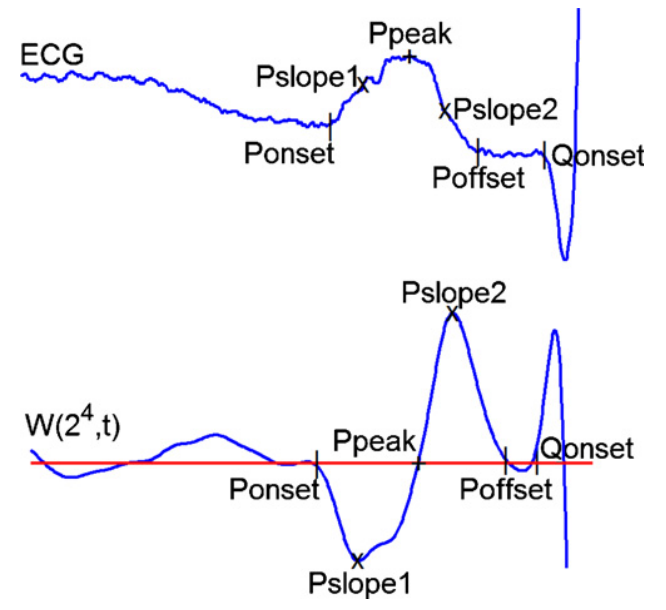

Figure 1. ECG segment of the $P$ wave and related fourth dyadic wavelet decomposition scale.

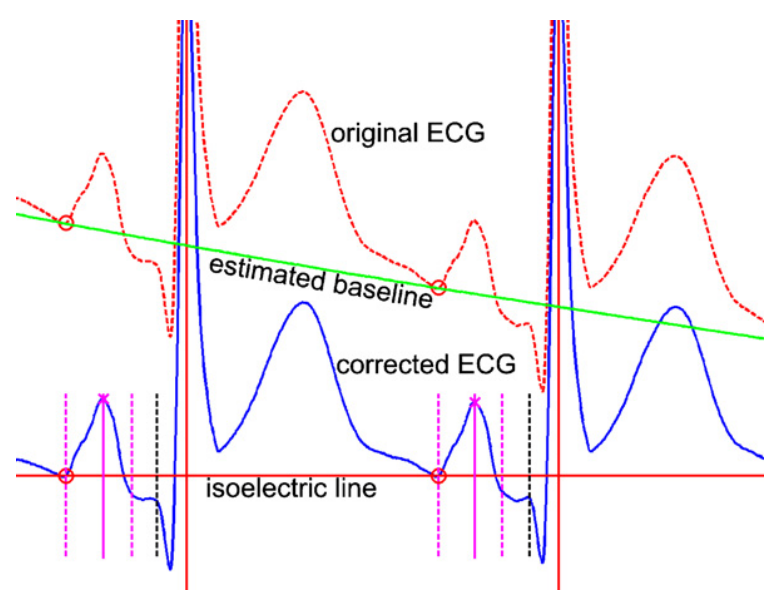

Figure 2. Example of the baseline specification and its correction for drift. Original ECG (dash line), estimated baseline drift (descending solid line), $P$ wave onset (circle), corrected ECG (solid line), isoelectric line (straight solid line).

The corrected ECG signal (without baseline drift) was obtained by subtracting the estimated baseline from the original ECG (with baseline drift) (figure 2).

\subsection{Measured features}

For each individual $P$ wave a vector consisting of 40 different measured features was determined and some of them are listed in table 4 . The mean value and the standard deviation of all measured features were calculated over all subsequent episodes of $15 \mathrm{~min}$ of the recorded data.

The signification of these prospectively selected features is as follows: the $P$ wave duration (PonPoff) reflects the propagation velocity of the atrial activation as well as atrial size. The duration of the first and second half of the $P$ wave (PonPpeak, PpeakPoff) indicates 
Table 4. Measured features.

\begin{tabular}{|c|c|}
\hline Feature & Description \\
\hline \multicolumn{2}{|l|}{ Time features } \\
\hline PonPoff & $P$ wave duration \\
\hline PonPpeak & First half of the $P$ wave duration \\
\hline PpeakPoff & Second half of the $P$ wave duration \\
\hline PoffQon & PQ segment duration \\
\hline PonQon & PQ interval duration \\
\hline PpeakRpeak & PR interval duration \\
\hline Pslope1Pslope2 & Pslope1-Pslope 2 interval duration \\
\hline $\mathrm{RR}, \mathrm{HR}$ & RR interval duration and heart rate \\
\hline \multicolumn{2}{|l|}{ Amplitude features } \\
\hline PQlevel & Level of the PQ segment \\
\hline Pamp, Ramp & $P$ wave and $R$ wave amplitude \\
\hline Qonamp & Level of the $Q$ wave onset (Qonset) \\
\hline Poffamp & Level of the $P$ wave ending (Poffset) \\
\hline \multicolumn{2}{|c|}{ Amplitude-time features } \\
\hline Pslope1 & Maximal positive slope of the $P$ wave \\
\hline Pslope2 & Maximal negative slope of the $P$ wave \\
\hline Aonoff & $P$ wave surface area \\
\hline relPslope1Pslope2 & Ratio of the maximal positive and negative slope \\
\hline \multicolumn{2}{|l|}{ Wavelet features } \\
\hline Wenergy & $P$ wave energy at five different wavelet scales \\
\hline relWenergy & Relative $P$ wave energy regarding to total \\
\hline Wentropy & Entropy, measure of the $P$ wave energy dispersion \\
\hline \multicolumn{2}{|c|}{ Normalized time features } \\
\hline PonPoff_RR & $P$ wave duration (normalized with RR) \\
\hline PonQon $R R$ & PQ interval duration (normalized with RR) \\
\hline PpeakRpeak_RR & PR interval duration (normalized with RR) \\
\hline \multicolumn{2}{|c|}{ Normalized amplitude features } \\
\hline relPQlevel_Pamp & Level of PQ segment (normalized with Pamp) \\
\hline relPQlevel_Ramp & Level of PQ segment (normalized with Ramp) \\
\hline relPamp_Ramp & Ratio between the amplitude of the $P$ wave and the $R$ wave \\
\hline
\end{tabular}

the symmetry of the $P$ wave shape. The duration of the PQ interval (PonQon) comprises the duration of atrial depolarization and the AV node delay up to the onset of ventricular depolarization.

Several timing features are correlated with the RR interval; therefore, all time features were normalized with the mean RR duration over the episode (PonPoff_RR, PonQon_RR, PpeakRpeak_RR).

The potential level of the PQ segment (PQlevel) was specified by the mean value of the signal in the interval between the ending of the $P$ wave (Poffset) and the beginning of the $Q$ wave (Qonset). It represents atrial repolarization and it is not electrically silent as is frequently stated (Ihara et al 2006). 
The maximal positive slope (Pslope1) and the maximal negative slope (Pslope2) of the $P$ wave were determined by using the wavelet transform. The wavelet coefficients of Pslope 1 and Pslope 2 are linearly proportional to the values of the ECG slopes expressed in $\mathrm{mV} / \mathrm{ms}$ units. The $P$ wave slopes depend on the propagation velocity of atrial impulses, the premature onset of atrial repolarization and the $P$ wave amplitude.

Wavelet decomposition of the ECG can be viewed as a filtering of the signal with the filter bank consisting of bandpass filters (wavelet scales) (Li et al 1995). Different parts of the spectrum contain different parts of the $P$ wave. Because of this, $P$ wave energy was measured at different wavelet scales (Wenergy), as was the ratio of the energy at each wavelet scale and the total $P$ wave energy (relWenergy). Entropy is a measure of $P$ wave energy dispersion at different parts of spectrum.

The heart position relative to the electrode position influences the value of all measured amplitude features. To compensate for this effect several amplitude features were normalized with respect to the $P$ wave or $R$ wave amplitude (relPQlevel_Pamp, relPQlevel_Ramp, relPamp_Ramp).

\subsection{Statistical analysis}

The complete data set comprised the data of 50 patients. For each patient, the $48 \mathrm{~h}$ ECG was divided into 15 min episodes, yielding a total of 192 episodes. For each episode, segment by segment, we conducted two sample $t$-tests $(p<0.05)$ for data normally distributed and the non-parametric Wilcoxon rank sum test (Mann-Whitney $U$-test, $p<0.05$ ) for non-normally distributed data. This test was used to test univariate group differences between the mean or median values over the individual episodes of the 40 measured variates.

For each of the features listed in table 4, over each of the 192 successive episodes the results of the statistical tests (independent two sample $t$-tests and Wilcoxon rank sum test) were scored as 1 if significance was observed, else as 0 . For each feature the cumulative score over the 192 episodes was inspected.

The probability density distribution (histogram) for all measured features was estimated, both for the AF group and the SR group. The level of the PQ segment in the AF group revealed a bimodal distribution, whereas the SR group exhibited a normal distribution (figure 5). Because of this, the statistical testing of this feature did not attain a significant difference in either the means or the medians of the groups. To resolve the controversy we have divided patients of the bimodal AF group into the two unimodal subgroups: the AF1 subgroup with the average level of the PQ segment lower than $-27 \mu \mathrm{V}$ and the AF2 subgroup with the average level higher than $-27 \mu \mathrm{V}$. Afterward, probability density functions for all three dominant predictors were estimated (figure 6). The univariate group difference between the SR group and individually AF1 and AF2 subgroups was tested again using the same methodology.

Here, we decided to test the application of two linear models based on Fisher linear discriminant analysis (FDA) and one nonlinear model of a classification tree for the AF prediction based on the most promising three features. The first, bivariate model (FDA1) included the RR interval and $P$ wave duration, and the second, trivariate model (FDA2) had an additional feature-level of the PQ segment.

Classification trees are based on the set of if-then logical univariate splits, which try to achieve the best possible prediction accuracy (Breiman 1993). Models based on a classification tree are non-parametric and nonlinear; therefore, they can reveal the nonmonotonic relationship between the variables performing multiple splits on the same variable (Sovilj et al 2007, 2006). The prediction model based on classification trees included three features: RR interval duration, $P$ wave duration and PQ segment level. 


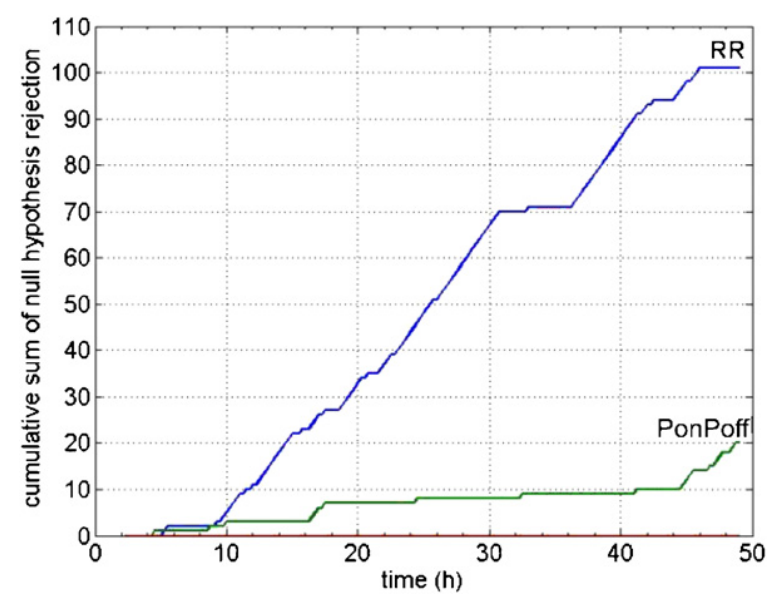

Figure 3. Cumulative score of null hypothesis rejection in time. Presented features were statistically significant in successive statistical tests: independent two sample $t$-tests $(p<0.05)$ for data normally distributed and non-parametric Wilcoxon rank sum test (Mann-Whitney $U$-test, $p<0.05$ ) for non-normally distributed data.
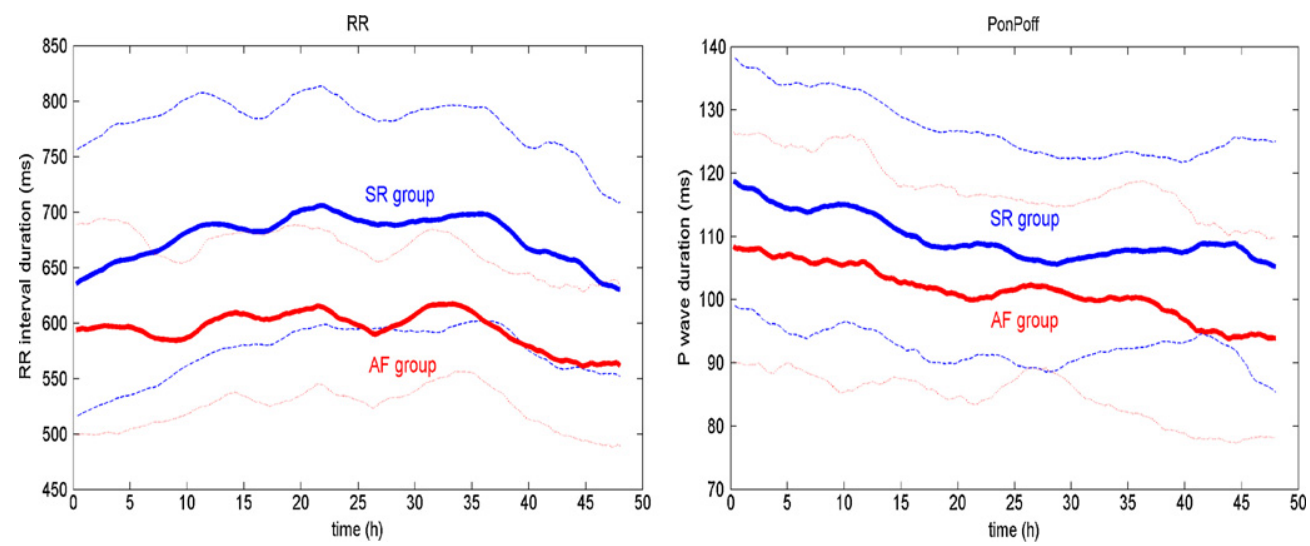

Figure 4. Trends of mean values of the RR interval and the $P$ wave duration over the $15 \mathrm{~min}$ episodes for the SR group (thick upper curve), the AF group (thick lower curve) for the time of $48 \mathrm{~h}$ after $\mathrm{CABG}$ and corresponding standard deviations for the SR group (dashed line) and AF group (dotted line).

\section{Results}

The results of statistical tests related to the two features that obtained the maximal cumulative score over the 192 episodes are shown in figure 3. All the other features tested remained well below the lower trace shown. In this way, the RR interval and $P$ wave duration were identified as the most promising univariate predictors of AF.

For the features that showed statistical significance (PonPoff and RR interval) a trend analysis of the mean values per group was performed (figure 4). The RR interval was found to be shorter in the AF group $(607 \pm 104 \mathrm{~ms}, 99 \pm 15 \mathrm{bpm})$ than in the SR group (678 \pm $77 \mathrm{~ms}, 88 \pm 9 \mathrm{bpm})$. The $P$ wave duration was also shorter in the AF group $(110 \pm 15 \mathrm{~ms})$ than in the SR group $(118 \pm 15 \mathrm{~ms})$. 


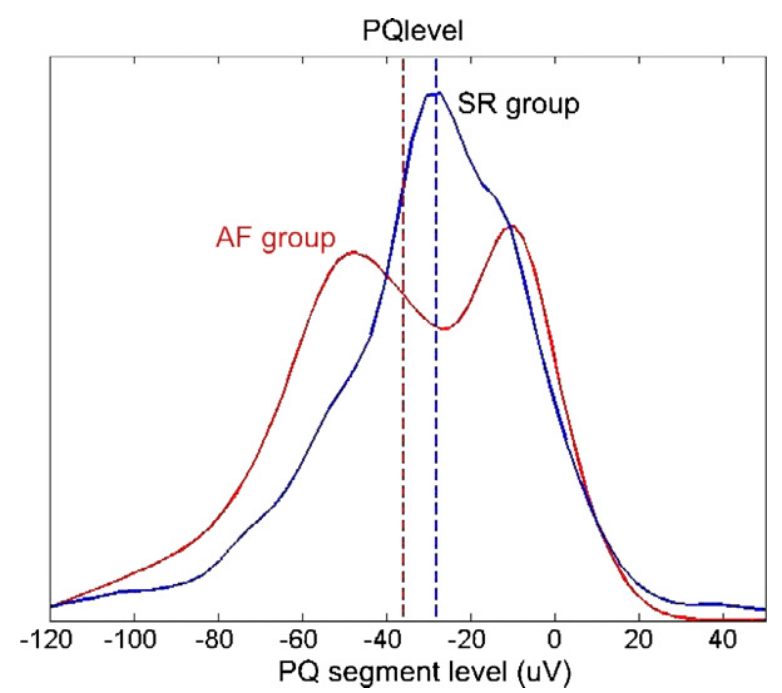

Figure 5. Estimation of probability density for the PQ segment level. AF group (bimodal distribution), SR group (normal distribution). Dashed lines mark the median values.

Table 5. Best prediction accuracy of Fisher linear discriminant models (FDA1 — bivariate, FDA2 tri-variate) and classification tree model.

\begin{tabular}{llll}
\hline & FDA1 & FDA2 & Classification tree \\
\hline Sensitivity & $72.0 \%$ & $74.2 \%$ & $84.8 \%$ \\
Specificity & $75.8 \%$ & $74.2 \%$ & $85.4 \%$ \\
Positive predictive value & $45.4 \%$ & $47.7 \%$ & $72.7 \%$ \\
Negative predictive value & $90.6 \%$ & $90.4 \%$ & $92.8 \%$ \\
Accuracy & $74.8 \%$ & $74.3 \%$ & $85.3 \%$ \\
\hline
\end{tabular}

In the standard statistical tests the PQ segment level did not show a significant difference in the mean or median values between groups. However, the inspection of estimated density distributions of both groups suggested clear differences based on a bimodal distribution observed in the AF group, compared to a unimodal distribution in the SR group (figures 5 and 6). The AF group showed two subgroups of patients (AF1 and AF2): one with a higher depression of the PQ segment level $(-52 \pm 18 \mu \mathrm{V})$ and the other with a higher elevation of the PQ segment level $(-7 \pm 9 \mu \mathrm{V})$, relative to the level in the SR group $(-29 \pm$ $30 \mu \mathrm{V})$. For the SR-AF1 group difference, heart rate and level of PQ segment are significant predictors and for the SR-AF2 group difference, all three predictors are significant: $P$ wave duration, heart rate and PQ segment level. The visualization of these differences can be seen in figure 6.

Deploying Fisher linear discriminant analysis, two multivariate linear models for the prediction of post-operative AF were tested. Both models, bivariate model (FDA1) and trivariate model (FDA2), demonstrated a monotonic rising trend in the prediction accuracy, and the best results are shown in table 5 .

This indicated that the inclusion of the PQ segment level did not increase the performance of the discrimination between the groups based on linear discrimination methods, as could be 

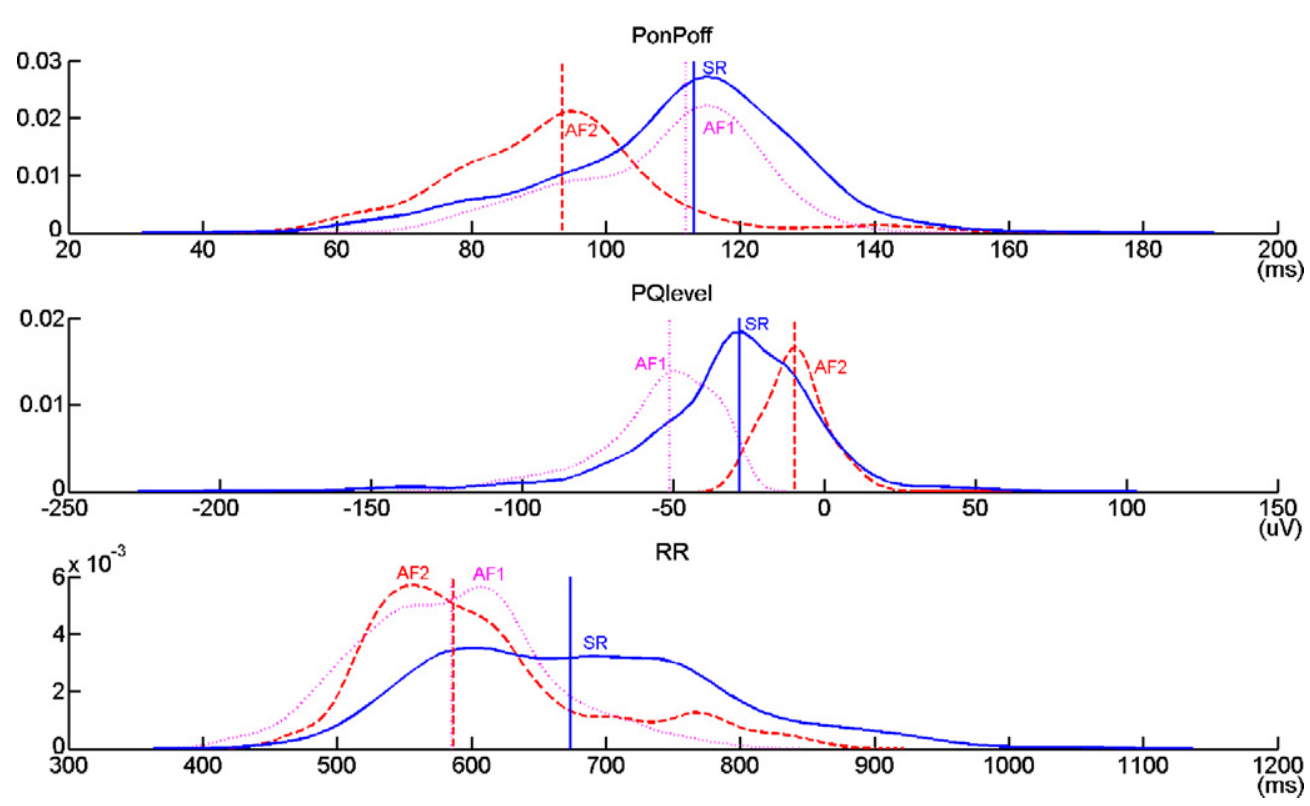

Figure 6. Probability density functions for three dominant predictors: $P$ wave duration (PonPoff), level of PQ segment (PQ level) and heart rate (RR), for SR group (solid line), AF1 subgroup (dotted line) and AF2 subgroup (dashed line). Vertical lines mark the median values for each group and subgroup.

expected in view of the results of the univariate test. However, the results shown in figure 5 did hold some promise for the usefulness of this feature. To study this, a nonlinear prediction method was tested.

The nonlinear prediction model based on a classification tree was trained on randomly selected samples (10\% of all data, training/learning sample) and subsequently tested on all individual segments in time from the beginning to the end of recording (testing sample; cross-validation). The resulting classification tree (figure 7), on the basis of the threshold levels identified, suggests that patients prone to developing AF have higher heart rate $(\mathrm{RR}<$ $621 \mathrm{~ms}$ ), greater depression of PQ segment level (PQ level $<-43 \mu \mathrm{V}$ ) or shorter $P$ wave duration $(<101 \mathrm{~ms})$.

When applied to the subsequent recorded episodes, the nonlinear model (figure 7) revealed a rising trend in the quality of the prediction (figure 8). Around the second day after CABG, when the AF incidence is most frequent, the prediction model becomes more and more accurate. At $48 \mathrm{~h}$ after the termination of CABG, the performance of the model was highest: average sensitivity $84.8 \%$, specificity $85.4 \%$, positive predictive value $72.7 \%$ and negative predictive value $92.8 \%$. The accuracy of the model was $85.3 \%$.

\section{Discussion}

The aim of this study is the development of a prediction model for the identification of patients at high risk of AF following CABG. Prediction of AF prior to the onset of AF could enable more effective prophylactic therapy. Timely administration of an anti-arrhythmic treatment to the patients as identified prone to AF (AF group) could decrease the incidence of arrhythmia. 


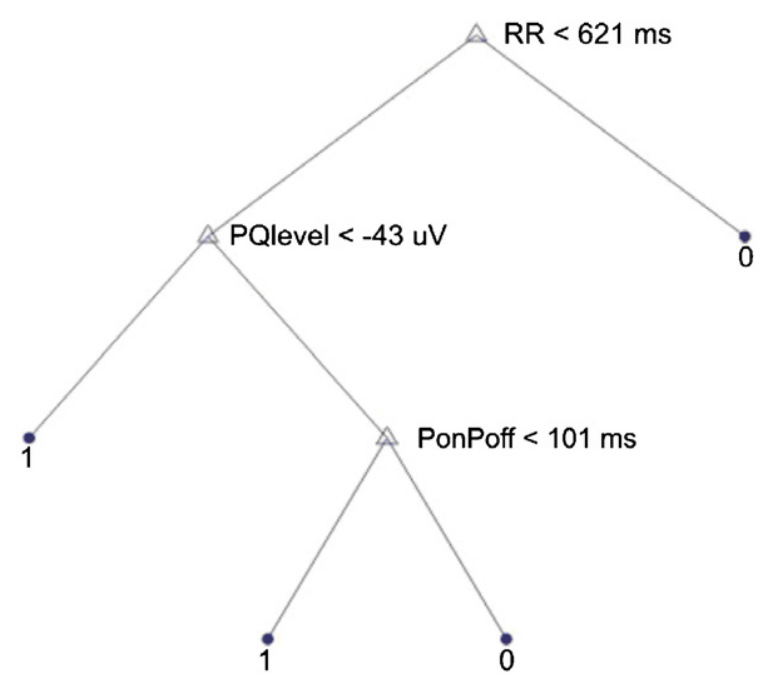

Figure 7. Prediction model based on a classification tree. Predictions are marked: $1=$ AF group, $0=\mathrm{SR}$ group. If a patient has a RR interval duration $<621 \mathrm{~ms}$ and a depressed PQ segment level $<-43 \mu \mathrm{V}$, then he/she is classified as prone to AF, or if a PQ segment level $>-43 \mu \mathrm{V}$ and $P$ wave duration $>101 \mathrm{~ms}$, then he/she is classified as prone to sinus rhythm, otherwise as prone to AF.
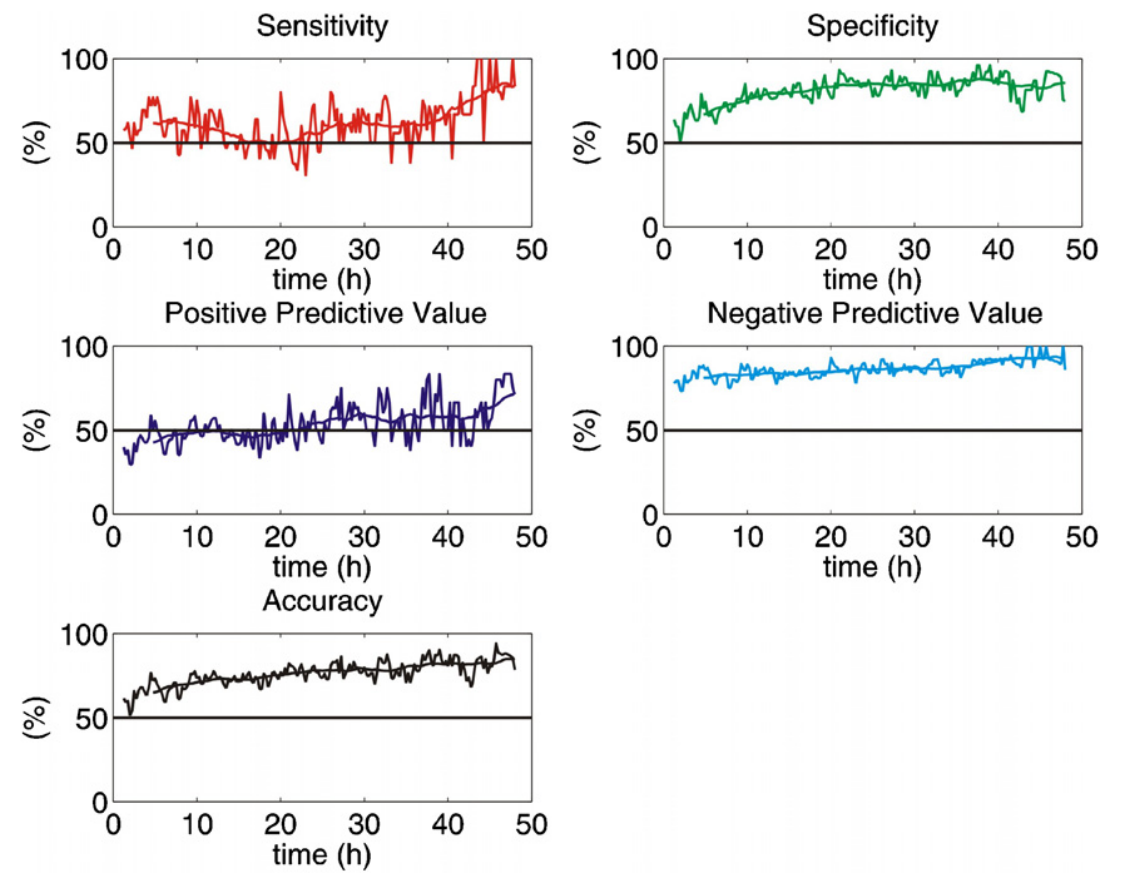

Figure 8. Prediction quality of the nonlinear method based on a classification tree over time, specified by sensitivity, specificity, positive predictive value, negative predictive value and accuracy (thin lines). The thick lines represent the trends smoothed by a moving average window (involving 16 points) as a more correct trend measure. 
Patients assessed as being resistant to AF and prone to maintaining sinus rhythm (SR group) could be spared from the side effects of drugs.

Prediction of post-operative AF derived from the ECG is usually based on signal averaged $P$ wave ECG (SAECG), standard ECG leads II or V1 and heart rate variability (HRV). Several studies have suggested that the duration of a filtered signal averaged $P$ wave can be an independent predictor of post-operative AF (Aytemir et al 1999, Caravelli et al 2002, Klein et al 1995, Stafford et al 1997, Steinberg et al 1993, Vassilikos et al 2003, Zaman et al 1997). However, no consensus was reached for the classification based on the $P$ wave prolongation, and stated threshold levels spanned the range from 122 to $155 \mathrm{~ms}$. The prediction accuracy was found to increase when the $P$ wave duration is combined with other risk factors: low left ventricular ejection fraction $(<40 \%)$ or low serum magnesium concentration $\left(<0.7 \mathrm{mmol} \mathrm{l}^{-1}\right)$ (Hakala and Hedman 2003).

A few studies have attempted to predict post-operative AF by measuring the $P$ wave duration in leads selected from the standard 12-lead ECG, typically lead II or precordial lead V1. The results are controversial. Observing just a single lead, some studies have found a significant difference in the $P$ wave duration and also a correlation with the SAECG $P$ wave duration (Aytemir et al 1999, Dimmer et al 1998a). The observations in more recent studies of Stafford et al (1997) and Caravelli et al (2002) in their analyses did not corroborate these results. Tiskouris et al (2001) found that the $P$ wave duration after CABG is not a constant in time; it alters and is greatest on the third day after $\mathrm{CABG}$, which coincides with the peak incidence of post-operative AF.

In general, the methods for measuring the $P$ wave duration are not unambiguously defined, nor the threshold levels for classing the $P$ wave as prolonged. Replication of the studies is difficult and their potential clinical use is unclear.

Heart rate variability has been analyzed in order to study a potential involvement of dysfunction of the autonomic heart rate control in inducing AF. It has been suggested that a heightened sympathetic or vagal tone might occur before the onset of AF. It has been demonstrated that the prediction is feasible just prior to the onset of AF. However this is too late for the application of an efficient prophylactic therapy (Dimmer et al 1998b, Hogue et al 1998).

Extended ECG monitoring, as used in this study, proved to be valuable in the development of methods for the prediction of post-operative AF. While recording just lead II of the standard 12-lead ECG only (demanding two electrodes only), the achieved quality of AF prediction (table 5) was comparable to the SAECG studies (table 2) in which the three orthogonal leads of vectorcardiography (ideally demanding seven electrodes) were involved (Aytemir et al 1999, Caravelli et al 2002, Klein et al 1995, Stafford et al 1997, Steinberg et al 1993, Vassilikos et al 2003, Zaman et al 1997). A more strict and faithful comparison of methods for post-operative AF prediction could be done in a project in a future by making our database available to other investigator groups.

Possible causes of higher heart rate in the post-operative process may be post-operative infection or inflammation of the atrium, fever, compensation for ischemia elsewhere, hypoxia, hypotension, hypovolemia and pericarditis. Nevertheless, the patients with inflammation markers, fever or pericarditis were excluded from the statistics.

It is assumed that a decreased propagation velocity of atrial impulses leads to prolongation of the $P$ wave duration. Our assumption is that a decreased refractory period and a decreased transmembrane potential duration cause premature atrial repolarization and as a result cause a change in the level of the PQ segment.

The observed $P$ wave duration in lead II is the result of overlapping between the two effects: (1) prolongation of the $P$ wave duration due to the decrease in the conduction velocity of atrium 
and (2) shortening of the $P$ wave duration due to overlapping between atrial depolarization and possible premature atrial repolarization, as a result of decreased refractory period. Since the patients prone to post-operative AF can manifest one, the other or both effects, it is difficult to assess which of the effects have a deeper impact on the $P$ wave duration observed in only one bipolar lead. We assume that in II lead the slow propagation velocity would prolong the $P$ wave duration, while the shortening of atrial refractory period would decrease the $P$ wave duration. The same phenomenon is likely to be the reason for the bimodal distribution of the PQ segment in the AF group as shown in figure 5 as well for the existence of the two AF subgroups AF1 and AF2.

According to our data we would summarize: (1) the AF1 subgroup of patients in comparison with the SR group has the $P$ wave duration in lead II 'normal' or the same as the SR group, higher depression of PQ segment level and higher heart rate; (2) the AF2 subgroup of patients in comparison with the SR group has a shorter $P$ wave duration, higher elevation of PQ segment level and also higher heart rate.

The prediction is based on three features: RR interval, $P$ wave duration and PQ segment level. The PQ segment lies between the $P$ wave ending and the $Q$ wave onset. The $Q$ wave onset is the beginning of ventricular depolarization and any atrial activity is masked afterward. As stated the PQ segment is not electrically silent as was assumed; rather it can contain a part of atrial repolarization toward new studies (Ihara et al 2006), particularly if the atrial repolarization is premature, which is the case in the patients prone to AF (Tranchesi et al 1960). The effectiveness of the inclusion of this novel feature was brought out by its inclusion in a nonlinear prediction model based on a classification tree. By using this method instead of Fischer's linear discriminant method, the maximum observed overall accuracy increased from $74.3 \%$ to $85.3 \%$.

We have noted that the $P$ wave morphology is very unstable and inconstant through time, in particular for the AF group. In some patients this abnormality was determined at the very beginning and was consistent through the entire time of assessment. For others within the AF group the detected abnormalities were more sporadic at the beginning and became more frequent subsequently. Therefore, the performance of the prediction was found to increase with time, reaching a maximum $48 \mathrm{~h}$ post-CABG (figure 8 ). This is also illustrated in figure 9, which depicts the observed trend of true positive rate (sensitivity) and false positive rate (1-specificity).

The time interval from when the abnormality was determined and the initiation of AF depends mainly on the desired accuracy level of the prediction model; if performed in the period 24-48 h after CABG, when the accuracy level of prediction is higher, it would be overdue for $3 / 14(21.4 \%)$ patients with AF developed in the preceding and current day, but it would also be punctual for $11 / 14$ (78.6\%) patients with AF developed in the following days. Thus, the results of our study suggest that risk assessment and post-operative AF prediction may be optimal when performed in the period from 24 to $48 \mathrm{~h}$ after CABG. In the preceding period, the mechanisms that induce post-operative AF possibly are not yet sufficiently developed.

AF prediction, in the period 24-48 h after CABG, meets the requirements for early AF prediction and leaves time for more effective prophylactic therapy. The presented nonlinear prediction model based on the classification tree method is intended for continuous monitoring, since the AF emerging process is non-stationary and dynamic in nature. Increased or decreased predictor values would classify a patient as prone or not prone to $\mathrm{AF}$, alarming in case monitored values of a particular patient reach preset threshold values of a classifier.

Further improvements that could cast aside the limitations of the method could be the inclusion of additional leads for more accurate measurement of the $P$ wave duration and PQ segment level, acquisition of series longer than $48 \mathrm{~h}$ after CABG, and the design and testing of 


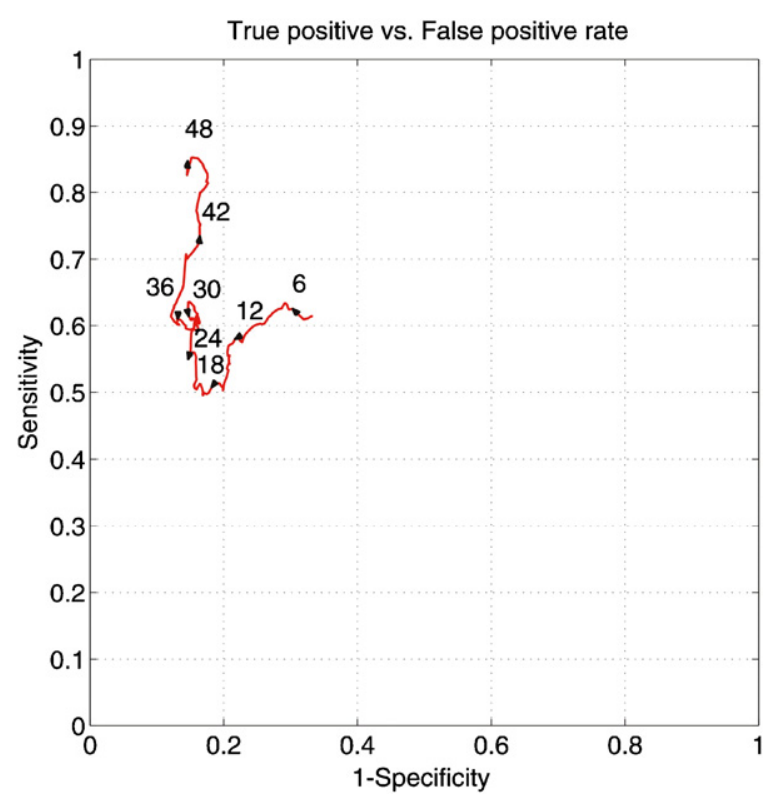

Figure 9. True positive versus false positive rate of the nonlinear prediction model based on a classification tree. The solid line shows an uprising trend in AF prediction quality with time. Arrows show the trend direction with time. The numbers next to the arrows represent the time in hours with reference to the termination of CABG.

other nonlinear prediction models which could further improve the prediction quality, covering to a greater extent the nonlinearity and non-stationarity of the underlying AF process.

\section{Acknowledgments}

This study was supported by the Ministry of Science, Education and Sport of the Republic of Croatia, under grant no 036-0362979-1554. SS was supported by a Swiss Government scholarship for a research study at the Swiss Federal Institute of Technology in Lausanne (Ecole Polytechnique Fédérale de Lausanne-EPFL).

\section{References}

Andrikopoulos G, Dilaveris P and Richter D 2000 Increased variance of $P$ wave duration on the electrocardiogram distinguishes patients with idiopathic paroxysmal atrial fibrillation Pacing Clin. Electrophysiol. 23 1127-32

Aytemir K, Aksoyek S and Ozer N 1999 Atrial fibrillation after coronary artery bypass surgery: $P$ wave signal averaged ECG, clinical and angiographic variables in risk assessment Int. J. Cardiol. 69 49-56

Aytemir K, Ozer N, Atalar E and Sade E 2000 P wave dispersion on 12-lead electrocardiography in patients with paroxysmal atrial fibrillation Pacing Clin. Electrophysiol. 23 1109-12

Borzak S, Tisdale J, Amin N, Goldberg D, Frank D, Padhi I and Higgins R 1998 Atrial fibrillation after bypass surgery: does the arrhythmia or the characteristics of the patients prolong hospital stay? Chest 113 1489-91

Breiman L 1993 Classification and Regression Trees (Boca Raton, FL: Chapman and Hall)

Buxton A and Josephson M 1981 The role of $P$ wave duration as a predictor of postoperative atrial arrhythmias Chest 80 68-73

Caravelli P, De Carlo M, Musumeci G, Tartarini G, Gherarducci G, Bortolotti U, Mariani M and Mariani M 2002 $P$-wave signal-averaged electrocardiogram predicts atrial fibrillation after coronary artery bypass grafting Ann. Noninvasive Electrocardiol. 7 198-203 
Chang C et al 1999 The role of $P$ wave in prediction of atrial fibrillation after coronary artery surgery Int. $J$. Cardiol. $68303-8$

Creswell L, Schuessler R, Rosenbloom M and Cox J 1993 Hazards of postoperative atrial arrhythmias Ann. Thorac. Surg. 56 539-49

Dilaveris P, Gialafos E and Sideris S 1998 Simple electrocardiographic markers for the prediction of paroxysmal idiopathic atrial fibrillation Am. Heart J. $135733-8$

Dimmer C, Jordaens L and Gjorgov N 1998a Analysis of the $P$ wave with signal averaging to assess risk of atrial fibrillation after coronary bypass surgery Cardiology 89 19-24

Dimmer C, Tavernier R, Gjorgov N, Van Nooten G, Clement D and Jordaens L 1998b Variations of autonomic tone preceding onset of atrial fibrillation after coronary artery bypass grafting Am. J. Cardiol. 82 22-5

Fukunami M, Yamada T, Ohmori M, Kumagai K, Umemoto K, Sakai A, Kondoh N, Minamino T and Hoki N 1991 Detection of patients at risk for paroxysmal atrial fibrillation during sinus rhythm by $P$ wave-triggered signal-averaged electrocardiogram Circulation 83 162-9

Hakala T and Hedman A 2003 Predicting the risk of atrial fibrillation after coronary artery bypass surgery Scand. Cardiovasc. J. 37 309-15

Hashimoto K, Ilstrup D and Schaff H 1991 Influence of clinical and hemodynamic variables on risk of supraventricular tachycardia after coronary artery bypass J. Thorac. Cardiovasc. Surg. 101 56-65

Hogue C, Domitrovich P, Stein P and Despotis G 1998 RR interval dynamics before atrial fibrillation in patients after coronary artery bypass graft surgery Circulation $\mathbf{9 8} 429-34$

Ihara Z, Van Oosterom A and Hoekema R 2006 Atrial repolarization as observable during PQ interval J. Electrocadiol. 39 290-7

Kannel W B, Wolfs P A, Benjamin E J and Levy D 1998 Prevalence,incidence, prognosis, and predisposing conditions for atrial fibrillation: population-based estimates Am. J. Cardiol. 82 2N-9N

Klein M, Loring Evans S, Blumberg S, Cataldo L and Bodenheimer M 1995 Use of $P$ wave-triggered, $P$ wave signal averaged electrocardiogram to predict atrial fibrillation after coronary artery bypass surgery Am. Heart J. $129895-901$

Li C, Zheng C and Tai C 1995 Detection of ECG characteristic points using wavelet transforms IEEE Trans. Biomed. Eng. 42 21-9

Passman R, Beshai J, Pavri B and Kimmel S 2001 Predicting post-coronary bypass surgery atrial arrhythmias from the preoperative electrocardiogram Am. Heart J. 142 806-10

Petrač D 1996 Fibrilacija atrija (Zagreb: Medicinska naklada) (in Croatian)

Sovilj S, Magjarevic R and Rajsman G 2007 Classification methods for atrial fibrillation prediction after CABG Medicon 2007 (Ljubljana, Slovenia) IFMBE Proc. vol 16 pp 46-9

Sovilj S, Rajsman G and Magjarevic R 2005 Continuous multiparameter monitoring of $P$ wave parameters after CABG using wavelet detector Proc. Computers in Cardiology 2005 (Lyon, France) vol 32 pp 945-8

Sovilj S, Rajsman G and Magjarevic R 2006 Multiparameter prediction model for atrial fibrillation after CABG Proc. Computers in Cardiology 2006 (Valencia, Spain) vol 33 pp 489-92

Stafford P, Kolvekar S, Cooper J, Fothergill J, Schlindwein F, deBono D, Spyt T and Garratt C 1997 Signal averaged $P$ wave compared with standard electrocardiography and echocardiography for prediction of atrial fibrillation after coronary bypass grafting Heart 77 417-22

Steinberg J, Zelenkofske S, Wong S, Gelernt M, Sciacca R and Menchavez E 1993 Value of the $P$ wave signal-averaged ECG for predicting atrial fibrillation after cardiac surgery Circulation 88 2618-22

Stewart S, Hart C L, Hole D L and McMurray J J 2001 Population prevalence, incidence, and predictors of atrial fibrillation in the renfrew/paisley study Heart 86 516-21

Tranchesi J, Adelardi V and de Olivera J 1960 Atrial repolarization-its importance in clinical electrocardiography Circulation 22 635-44

Tsikouris J, Kluger J, Song $\mathrm{J}$ and White C 2001 Changes in $P$-wave dispersion and $P$-wave duration after open heart surgery are associated with the peak incidence of atrial fibrillation Heart Lung J. Acute Crit. Care 30 466-71

Vassilikos V, Dakos G and Chouvarda I 2003 Can $P$ wave wavelet analysis predict atrial fibrillation after coronary artery bypass grafting? Pacing Clin Electrophysiol. 26 305-9

Zaman A, Alamgir F, Richens T, Williams R, Rothman M and Mills P G 1997 The role of signal averaged $P$ wave duration and serum magnesium as a combined predictor of atrial fibrillation after elective coronary artery bypass surgery Heart 77 527-31 